Rationalizable Strategic Behavior and the Problem of Perfection

Author(s): David G. Pearce

Source: Econometrica, Vol. 52, No. 4 (Jul., 1984), pp. 1029-1050

Published by: The Econometric Society

Stable URL: http://www.jstor.org/stable/1911197

Accessed: 10/12/2010 20:32

Your use of the JSTOR archive indicates your acceptance of JSTOR's Terms and Conditions of Use, available at http://www.jstor.org/page/info/about/policies/terms.jsp. JSTOR's Terms and Conditions of Use provides, in part, that unless you have obtained prior permission, you may not download an entire issue of a journal or multiple copies of articles, and you may use content in the JSTOR archive only for your personal, non-commercial use.

Please contact the publisher regarding any further use of this work. Publisher contact information may be obtained at http://www.jstor.org/action/showPublisher?publisherCode=econosoc.

Each copy of any part of a JSTOR transmission must contain the same copyright notice that appears on the screen or printed page of such transmission.

JSTOR is a not-for-profit service that helps scholars, researchers, and students discover, use, and build upon a wide range of content in a trusted digital archive. We use information technology and tools to increase productivity and facilitate new forms of scholarship. For more information about JSTOR, please contact support@jstor.org. 


\title{
RATIONALIZABLE STRATEGIC BEHAVIOR AND THE PROBLEM OF PERFECTION
}

\author{
By David G. Pearce ${ }^{1}$
}

\begin{abstract}
This paper explores the fundamental problem of what can be inferred about the outcome of a noncooperative game, from the rationality of the players and from the information they possess. The answer is summarized in a solution concept called rationalizability. Strategy profiles that are rationalizable are not always Nash equilibria; conversely, the information in an extensive form game often allows certain "unreasonable" Nash equilibria to be excluded from the set of rationalizable profiles. A stronger form of rationalizability is appropriate if players are known to be not merely "rational" but also "cautious."
\end{abstract}

\section{INTRODUCTION}

"WhAT CONSTITUTES RATIONAL BEHAVIOR in a noncooperative strategic situation?" This paper explores the issue in the context of a wide class of finite noncooperative games in extensive form. The traditional answer relies heavily upon the idea of Nash equilibrium (Nash [17]). The position developed here, however, is that as a criterion for judging a profile of strategies to be "reasonable" choices for players in a game, the Nash equilibrium property is neither necessary nor sufficient. Some Nash equilibria are intuitively unreasonable, and not all reasonable strategy profiles are Nash equilibria.

The fact that a Nash equilibrium can be intuitively unattractive is well-known: the equilibrium may be "imperfect." Introduced into the literature by Selten [20], the idea of imperfect equilibria has prompted game theorists to search for a narrower definition of equilibrium. While this research, some of which will be discussed here, has been extremely instructive, it remains inconclusive. Theorists often agree about what should happen in particular games, but to capture this intuition in a general solution concept has proved to be very difficult. If this paper is successful it should make some progress in that direction.

The other side of the coin has received less scrutiny. Can all non-Nash profiles really be excluded on logical grounds? I believe not. The standard justifications for considering only Nash profiles are circular in nature, or make gratuitous assumptions about players' decision criteria or beliefs. The following discussion of these points is extremely brief, due to space constraints; more detailed arguments may be found in Pearce [18].

I am concerned here with situations in which players are unable to communi-

\footnotetext{
'I am very grateful to Bob Anderson and Hugo Sonnenschein for their invaluable assistance. Not everyone who commented on this work can be mentioned here, but I would particularly like to thank Mark Bagnoli, Doug Bernheim, Bentley MacLeod, John C. Harsanyi, Vijay Krishna, Roger Myerson, Robert Wilson, and the anonymous referees for their helpful suggestions. Finally, I wish to acknowledge a major intellectual debt that I owe my colleague, Dilip Abreu. Our countless discussions on game theory have played a central role in shaping my ideas about strategic behavior. Of course, only I can be held responsible for the statements made herein.
} 
cate with one another before or during the game. The most sweeping (and, perhaps, historically the most frequently invoked) case for Nash equilibrium theory in such circumstances asserts that a player's strategy must be a best response to those selected by other players, because he can deduce what those strategies are. Player $i$ can figure out $j$ 's strategic choice by merely imagining himself in $j$ 's position. But this takes for granted that there is a unique rational choice for $j$ to make; this uniqueness is not derived from fundamental rationality postulates, but is simply assumed. Furthermore, any argument suggesting that player rationality, combined with the structural characteristics of a game, inevitably renders all but one outcome "impossible," leads to conclusions that contradict widely accepted notions of "perfection" (Pearce [19]). Once one admits the possibility that a player may have several strategies that he could reasonably use, expectations may be mismatched. Player $i$ 's strategy will then be a best response to his (possibly incorrect) conjecture about others' strategies, not the actual strategies employed.

A less ambitious defense of Nash equilibrium is that although equilibrium might not be attained in a one-shot game, players will eventually arrive at some Nash profile if the game is repeated indefinitely. Among the many objections to this claim, the most conclusive is that there may well be supergame equilibria involving phenomena (implicit collusion, maintenance of reputation, and so on) that are incompatible with single-period maximizing behavior. It is misleading, then, to study a repeated game by investigating the Nash equilibria of the one-shot game. But a more persuasive story can be told in which different players are involved at each iteration of the game. Each player is concerned only with one-period payoffs, but can look to the history of play for guidance regarding the likely choices of his opponents. While one cannot prove that each generation of players will follow a pattern set by previous participants, such an outcome seems quite plausible. But we are interested in analyzing many situations for which no precedents exist (such as nuclear wars between superpowers) or in which continual changes in relevant variables (technological breakthroughs, new legislation, and so on) preclude prediction based on tradition. It then becomes crucial to understand precisely what are the implications of players' information and rationality.

Most of this paper is devoted to the development and evaluation of a solution concept called "rationalizability." 2 It is offered as an answer to my opening question: "What constitutes rational behavior in a noncooperative strategic situation?" No attempt is made to single out a unique strategy profile for each game; instead, a profile is rationalizable if each player has selected any strategy

\footnotetext{
${ }^{2}$ Rationalizability in normal form games was developed independently by Doug Bernheim [2]. The expression "ex ante equilibrium" which I used in earlier work [18] has been adandoned here in favor of Bernheim's descriptive term "rationalizability," in order to unify the terminology in the literature. Our papers are complementary in many respects, his analyzing more general games in normal form and comparing Nash equilibrium to rationalizability, and mine spending more time than his on the extensive form and problems of perfection.
} 
that is "reasonable" in a sense to be made precise. A single player might have many such strategies.

While allowing for more flexibility than the Nash solution concept permits, one wishes to eliminate the problem of imperfection. This is complicated by the fact that there are actually two types of behavior that have been labelled "imperfect" in the literature. The first involves "implausible behavior at unreached information sets" and arises only in games having some sequential nature. The second is intimately related to the first, but can occur even in perfectly simultaneous games. It concerns the taking of risks that seem "likely" to be costly, when there are no offsetting advantages for a player to consider. The first type of imperfection can be ruled out on the basis of rather innocuous rationality postulates. Elimination of the second type, however, requires an additional assumption, amounting to the assertion that players will exercise prudence when it is costless to do so. Accordingly, I define two solution concepts. The first, rationalizability, relies upon little more than logical deduction, and ignores the second type of imperfect behavior. A narrower solution concept, which I call cautious rationalizability, makes the additional assumption needed to eliminate imperfections of the second type.

For expositional purposes the early sections of the paper deal only with normal form representations of games. Because I believe that the additional structure provided by the extensive form is often important in determining how players will act, I interpret a normal form game as a convenient representation of a perfectly simultaneous game, in which no one can observe any move of any other player before moving himself. Such games can be analyzed without the encumbrance of the extensive form structure. The analysis of Sections 2 and 3 should be understood as an investigation of a special class of extensive form games. Indeed, the general solution concepts ultimately proposed in Sections 4 and 5 reduce to those of Sections 2 and 3 for nonstochastic games in which everyone moves simultaneously. Many of the central themes of the paper come across more clearly in these special games.

\section{RATIONALIZABILITY IN NORMAL FORM GAMES}

The purpose of this section is to develop a solution concept for finite normal form games, based on three assumptions:

Assumption (A1): When a player lacks an objective probability distribution over another player's choice of strategy, he forms a subjective prior that does not contradict any of the information at his disposal.

Assumption (A2): Each player maximizes his expected utility relative to his subjective priors regarding the strategic choices of others.

Assumption (A3): The structure of the game (including all participants' 
strategies and payoffs, and the fact that each player satisfies Assumptions (A1) and (A2)) is common knowledge (see Aumann [1]). Roughly speaking, some information $\theta$ is common knowledge if for any players $i, j, \ldots, k$, the statement " $i$ knows that $j$ knows that ... that $k$ knows $\theta$ " is true.

An $N$-person noncooperative normal form game

$$
G=\left(S^{1}, \ldots, S^{N} ; U^{1}, \ldots, U^{N}\right)
$$

is completely characterized by the finite nonempty sets $S^{i}$ of pure strategies, and real-valued utility functions $U^{i}$ having domain $\prod_{r=1}^{N} S^{r}$. The set $M^{i}$ of mixed strategies for player $i$ is a simplex in Euclidean space; $U^{i}$ is extended to $\prod_{r=1}^{N} M^{r}$ by an expected utility calculation. Let $M=\left(M^{1}, \ldots, M^{N}\right)$.

A strategy $\sigma \in M^{i}$ is strongly dominated if $\exists \gamma \in M^{i}$ such that $\forall\left(m^{1}, \ldots, m^{N}\right)$ $\in \prod_{r=1}^{N} M^{r}$,

$$
\begin{aligned}
& U^{i}\left(m^{1}, \ldots, m^{i-1}, \gamma, m^{i+1}, \ldots, m^{N}\right) \\
& \quad>U^{i}\left(m^{1}, \ldots, m^{i-1}, \sigma, m^{i+1}, \ldots, m^{N}\right) .
\end{aligned}
$$

A strategy $b \in M^{i}$ is a best response for $i$ to a profile $\left(m^{1}, \ldots, m^{N}\right)$ if $\forall d \in M^{i}$,

$$
\begin{aligned}
& U^{i}\left(m^{1}, \ldots, m^{i-1}, b, m^{i+1}, \ldots, m^{N}\right) \\
& \quad \geq U^{i}\left(m^{1}, \ldots, m^{i-1}, d, m^{i+1}, \ldots, m^{N}\right) .
\end{aligned}
$$

If $b \in B^{i} \subseteq M^{i}$ and instead the above weak inequality holds for every $d \in B^{i}$, then $b$ is a best response in $B^{i}$ to $\left(m^{1}, \ldots, m^{N}\right)$. Throughout the paper $\bar{A}$ denotes the convex hull of a set $A$. If $A \subseteq M^{i}$, a conjecture over $A$ can be regarded (for the purposes of expected utility calculations) as an element of $\bar{A}$ (see Lemma 1 and Lemma 2 of Appendix A).

I now define functions $R^{i}$ which, when applied to the vector $M$ of mixed strategy sets, yield the sets of "rationalizable" strategies for each player. Immediately following this definition is a discussion of its motivation.

DeFINITION 1: For arbitrary sets $H^{i} \subseteq M^{i}, i=1, \ldots, N$, let $H^{i}(0)=H^{i}$, and for each $i$ define $H^{i}(t)$ inductively for $t=1,2, \ldots$ by $H^{i}(t)=\left\{\alpha \in H^{i}(t-1)\right.$ : $\exists \gamma \in \prod_{r=1}^{N} \bar{H}^{r}(t-1)$ such that $\alpha$ is a best response in $H^{i}(t-1)$ to $\left.\gamma\right\}$.

Define

$$
R^{i}\left(H^{1}, \ldots, H^{N}\right)=\bigcap_{t=1}^{\infty} H^{i}(t) .
$$

Thus the operation $R^{i}$ is an iterative procedure; at each stage, a strategy is retained only if it is a best response to some conjecture over strategies (for other players) that have not been removed at an earlier stage. By Assumptions (A1) 
and (A2) each player chooses a best response to some strategy $\left(\beta^{1}, \ldots, \beta^{N}\right)$ $\in \prod_{r=1}^{N} M^{r}$; in the notation of Definition 1, i's strategic choice lies in $M^{i}(1)$. Since this is an implication of Assumptions (A1) and (A2), which by (A3) are common knowledge, each player knows this information, and restricts his conjecture to elements of $\prod_{r=1}^{N} \bar{M}^{r}(1)$. Thus a best response of any player $j$ to his conjecture, is an element of $M^{j}(2)$. Again this is common knowledge, and $t$-fold iteration of this argument, for any $t$, establishes that strategic choices lie within the sets $M^{1}(t), \ldots, M^{N}(t)$. This being true for all $t$, players restrict themselves to the sets $R^{1}(M), \ldots, R^{N}(M)$.

Can we exclude any other strategies on the basis of Assumptions (A1), (A2), and (A3)? Proposition 1 below makes it evident that the vector $\left(R^{1}(M), \ldots\right.$, $R^{N}(M)$ ) has the best response property:

Definition 2: For sets $A^{i} \subseteq M^{i}, i=1, \ldots, N,\left(A^{1}, \ldots, A^{N}\right)$ has the best response property if $\forall i, \sigma \in A^{i}$ implies $\exists \gamma \in \prod_{r=1}^{N} \bar{A}^{r}$ such that $\sigma$ is a best response to $\gamma$.

This means that if player $i$ chooses $\alpha$, he can "justify" his choice by explaining that $\alpha$ is a best response to some $\left(\gamma^{1}, \ldots, \gamma^{N}\right) \in \prod_{r=1}^{N} \bar{R}^{r}(M)$. Moreover, $i$ 's guess about what any other player $j$ is doing is also reasonable, in the sense that $\gamma^{j}$ can be expressed as a convex combination of strategies in $R^{j}(M)$, which are themselves best responses to conjectures that $j$ might make about other players' strategies. The latter conjectures are in turn "justified" by the existence of further strategy profiles in $\prod_{r=1}^{N} R^{r}(M)$, and so on. Thus, for any strategy $\alpha \in R^{i}(M)$, there is an infinite succession of conjectures, each of them consistent with Assumptions (A1), (A2), and (A3), "rationalizing" the choice of $\alpha$. This motivates the formal definition of the solution concept.

Definition 3: Given a finite game $G=\left(S^{1}, \ldots, S^{N} ; U^{1}, \ldots, U^{N}\right)$ with the vector $M$ of associated mixed strategy sets, the set of rationalizable strategies for player $i$ is $R^{i}(M)$. A profile $\left(\alpha^{1}, \ldots, \alpha^{N}\right)$ is rationalizable if $\alpha^{i} \in R^{i}(M) \forall i$.

In order to state the main results of this section, an additional definition is necessary.

Definition 4: $A \subseteq M^{i}$ has the pure strategy property if $\alpha \in A$ implies that every pure strategy given positive weight by $\alpha$ is also in $A$.

Proposition 1: If $H^{i} \subseteq M^{i}$ and $H^{i}$ is closed, nonempty, and satisfies the pure strategy property, $i=1, \ldots, N$, then (a) $H^{i}(t)$ is closed, nonempty, and satisfies the pure strategy property $\forall i$, and $t=1,2, \ldots ;$ (b) for some integer $k, H^{i}(t)=H^{i}(k)$ for all $t \geq k, i=1, \ldots, N$.

Proof: Proposition 1 is a special case of Proposition 4, proved in Section 4. It is clear from (b) that $\left(R^{1}(M), \ldots, R^{N}(M)\right)$ has the best response property. 
COROLlaRY: For each player $i$, the set of rationalizable strategies $R^{i}(M)$ is nonempty, and in fact contains at least one pure strategy.

Proof: Set $\left(H^{1}, \ldots, H^{N}\right)=\left(M^{1}, \ldots, M^{N}\right)$ in Proposition 1.

The need for players to randomize in many Nash equilibria has long been considered somewhat puzzling (see, for example, the discussion in Luce and Raiffa [14, pp. 74-76]. The incentive for randomization seems to be the need to "evade" one's opponents. But in the present context, opponents are not always able to figure out a player's strategic choice; such a player can hide without randomizing, camouflaged by the uncertainty of the other players.

The following definition and proposition provide an illuminating characterization of the rationalizable sets, without recourse to any iterative procedure.

Definition 5: For each $i$, define

$$
\begin{aligned}
E^{i}= & \left\{x \in M^{i}: \exists X^{1}, \ldots, X^{N}\right. \text { with the best response property, } \\
& \text { and } \left.x \in X^{i}\right\} .
\end{aligned}
$$

Proposition 2: $E^{i}=R^{i}(M) \forall i$.

Proof: Since $\left(R^{1}(M), \ldots, R^{N}(M)\right)$ satisfies the best response property, $R^{i}(M) \subseteq E^{i} \forall i$, by definition. To establish the converse, note first that $\left(E^{1}, \ldots, E^{N}\right)$ has the best response property. $\alpha \in E^{i}$ implies $\exists X^{1}, \ldots, X^{N}$ such that $\alpha \in X^{i}$ and $\alpha$ is a best response to some $\gamma \in \prod_{r=1}^{N} \bar{X}^{r}$. But $\gamma \in \prod_{r=1}^{N} \bar{E}^{r}$ since $X^{r} \subseteq E^{r} \forall r$. Thus $E^{1}, \ldots, E^{N}$ have the best response property, which implies $E^{i} \subseteq M^{i}(1) \forall i$ (see Definition 1). An inductive argument completes the proof: assume that for some $t, E^{i} \subseteq M^{i}(t) \forall i$. Then $\alpha \in E^{i}$ implies $\alpha$ is a best response to some $\gamma \in \prod_{r=1}^{N} \bar{M}^{r}(t)$, and hence $\alpha \in M^{i}(t+1)$. Thus for all $t$ and $i$, $E^{i} \subseteq M^{i}(t)$, therefore $E^{i} \subseteq R^{i}(M) \forall i$.

Corollary: If $\left(n^{1}, \ldots, n^{N}\right)$ is a Nash equilibrium, $\left(n^{1}, \ldots, n^{N}\right)$ is rationalizable.

Proof: $\left(\left\{n^{1}\right\}, \ldots,\left\{n^{N}\right\}\right)$ has the best response property, so $n^{i} \in E^{i}$ $=R^{i}(M) \forall i$.

Since a Nash equilibrium always exists for finite games (Nash [17]), this furnishes an alternative proof that the rationalizable sets are nonempty.

Bernheim's definition of a rationalizable strategy makes explicit use of "belief systems." Apart from the fact that his definition applies to a more general class of strategy spaces, it is equivalent to Definitions 3 and 5 above; this is the content of his Proposition 3.2 (Bernheim [2]). 
In 2-person games, a strategy is strongly dominated if and only if there is no conjecture to which the strategy is a best response (see Appendix B, Lemma 3). Hence for 2-person games, rationalizable strategies are those remaining after the iterative deletion of strongly dominated strategies. ${ }^{3}$ This does not hold for $N \geq 3$, where the rationalizable sets may be strictly smaller than (but always contained in) those resulting from the iterative removal of dominated strategies; an example of this strict containment is given by Pearce [18, p. 17]. Proofs of the equivalence of the two procedures for $N=2$ could easily be extended to arbitrary $N$ if a player's opponents could coordinate their randomized strategic actions.

The matrix game $G_{1}$ below provides a simple example in which non-Nash profiles are rationalizable. The reader can easily verify that $\left(\alpha_{1} ; \beta_{1}\right)$ is a Nash equilibrium that Pareto dominates all other Nash equilibria of $G_{1}$. Some game theorists, then, would single out $\left(\alpha_{1} ; \beta_{1}\right)$ as the solution of $G_{1}$. Opposition is bound to come from others who would insist that in the face of 1's indifference between $\alpha_{1}$ and $\alpha_{2}$ (regardless of 2's strategic choice), 2 should consider it equally likely (according to the principle of insufficient reason) that $\alpha_{1}$ and $\alpha_{2}$ will be played. 2 would then choose $\beta_{2}$, which is not his strategy in the Pareto dominant equilibrium. In a case such as this where two attractive rules of thumb conflict with one another, should we be astonished if 1 decides to play $\alpha_{1}$, for example, while 2 plays $\beta_{2}$ ? The profile is clearly rationalizable (as is every profile in $G_{1}$ ), but not a Nash equilibrium.

The principal drawback of rationalizability is clear: it typically does not allow a specific prediction to be made about strategic choice. (For example, in the game "matching pennies," all strategies are rationalizable.) But this indeterminacy is an accurate reflection of the difficult situation faced by players in a game. The rules of a game and its numerical data are seldom sufficient for logical deduction alone to single out a unique choice of strategy for each player. To do so one requires either richer information (such as institutional detail or perhaps historical precedent for a certain type of behavior) or bolder assumptions about how players choose strategies. Putting further restrictions on strategic choice is a complex and treacherous task. But one's intuition frequently points to patterns of behavior that cannot be isolated on the grounds of consistency alone. Formalizing this intuition in specific solution concepts would seem to be a matter of high priority; I interpret papers such as Harsanyi [11] to be in this spirit.

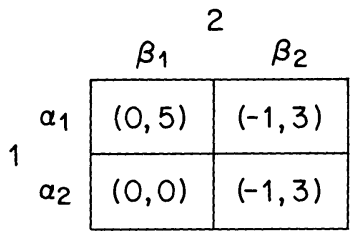
$G_{1}$

\footnotetext{
${ }^{3}$ Such procedures have long been a part of the game-theoretic literature; see for example Gale [8], Farquharson [6], and Luce and Raiffa [14], as well as the more recent work by Moulin [15].
} 


\section{CAUTIOUS RATIONALIZABILITY IN THE NORMAL FORM}

The notion of an imperfect equilibrium was originally conceived (see Selten [20]), and is still most commonly perceived, as a problem arising because of "implausible behavior at unreached information sets." This is obviously applicable only to extensive form games, which are treated in later sections. But a related phenomenon appears in normal form games, and has received some attention. In particular the paper by Myerson [16] on perfect and proper equilibria concerns exactly this issue.

Myerson's opening example is perhaps the simplest illustration of the problem at hand. $G_{2}$ has two Nash equilibria. In the first, 1 and 2 select the pure strategies $\alpha_{1}$ and $\beta_{1}$ respectively. In the second, they choose $\alpha_{2}$ and $\beta_{2}$ respectively. The latter equilibrium is, as Myerson indicates, counterintuitive: "it would be unreasonable to predict $\left(\alpha_{2}, \beta_{2}\right)$ as the outcome of the game. If player 1 thought that there was any chance of player 2 using $\beta_{1}$, then 1 would certainly prefer $\alpha_{1}$ " (Myerson [16, page 74]). It is clear that 1 is taking an unnecessary risk by choosing $\alpha_{2}$. He has nothing to gain by doing so, and possibly something to lose. The same applies to player 2, who would be foolish to choose $\beta_{2}$.

Explanations of why a certain equilibrium is to be considered "imperfect" usually involve stories about players making mistakes with small positive probabilities. This is a departure from tradition in the theory of games, and one senses a certain reluctance in Selten's remarks: "There cannot be any mistakes if the players are absolutely rational. Nevertheless, a satisfactory interpretation of equilibrium points in extensive games seems to require that the possibility of mistakes is not completely excluded. This can be achieved by a point of view which looks at complete rationality as a limiting case of incomplete rationality" (Selten [21, Section 7]). The same reasoning is employed in normal form games, and Myerson concludes his commentary on the game $G_{2}$ by saying that "... there is always a small chance that any strategy might be chosen, if only by mistake. So in our example, $\alpha_{1}$ and $\beta_{1}$ must always get at least an infinitesimal probability weight, which will eliminate $\left(\alpha_{2}, \beta_{2}\right)$ from the class of perfect (and proper) equilibria" (Myerson [16, p. 74]).

In my opinion the "slight mistakes" story does not do justice to our intuition about how players make their decisions. In game $G_{2}$, if 1 prefers $\alpha_{1}$ to $\alpha_{2}$, it is not because he believes that 2 might "make a mistake" and play $\beta_{1}$. On the contrary, $\beta_{1}$ would be an eminently reasonable choice for 2 (regardless of 1's choice). 1's reluctance to choose $\alpha_{2}$ reflects 1's belief that 2 is likely to choose $\beta_{1}$ deliberately, not as a result of incomplete rationality. Similarly, 2 is likely to use $\beta_{1}$ because he expects that 1 will probably select $\alpha_{1}$; no errors enter the picture. I will argue that

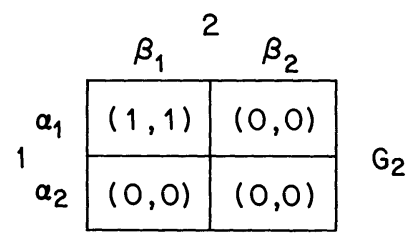


there is no need to base an analysis of imperfect behavior on incomplete rationality; an alternative is available which conforms more closely to intuition. First, an extremely brief sketch of the solution concepts proposed by Selten and Myerson is given. This is not meant to be a substitute for reading the original definitions.

In a game $G=\left(S^{1}, \ldots, S^{N} ; U^{1}, \ldots, U^{N}\right)$, a totally mixed strategy for player $i$ is a mixed strategy giving positive weight to each pure strategy in $S^{i}$. For any small positive number $\epsilon$, an $\epsilon$-equilibrium of $G$ is a profile of totally mixed strategies $\left(t^{1}, \ldots, t^{N}\right)$ such that for each $i$, player $i$ gives weight greater than $\epsilon$ to a given element $s$ of $S^{i}$ only if $s$ is a best response to $\left(t^{1}, \ldots, t^{N}\right)$. If $\left(z^{1}, \ldots, z^{N}\right)$ is the limit of $\epsilon$-equilibria as $\epsilon \rightarrow 0,\left(z^{1}, \ldots, z^{N}\right)$ is said to be a perfect equilibrium of $G$. (Each component of $\left(t^{1}, \ldots, t^{N}\right)$ is an element of Euclidean space; convergence is with respect to the usual Euclidean metric.) This is Myerson's formulation (Myerson [16]) of what is often called "trembling hand perfect equilibrium," originally defined by Selten [21] on the extensive form.

Roughly speaking, an $\epsilon$-proper equilibrium is a "combination of totally mixed strategies in which every player is giving his better responses much more probability weight than his worse responses (by a factor $1 / \epsilon)$, whether or not those 'better' responses are 'best' ... . We now define a proper equilibrium to be any limit of $\epsilon$-proper equilibria" (Myerson [16, p. 78]).

Requiring, as proper equilibrium does, that when contemplating an opponent's "trembles," a player should give much higher weight to relatively innocuous mistakes than to those which would cause the opponent serious damage, suggests that one is interested in "sensible trembles." In other words, the idea behind proper equilibrium seems to be that a player should be open-minded about various reasonable alternative strategies his opponents might use; the random component attributed to an opponent's action must not be arbitrary. While it is important to insist that doubts entertained by a player regarding his opponents' strategies should be concentrated upon reasonable possibilities, proper equilibrium attempts to enforce this without reference to any theory specifying what possibilities are realistic. This explains the failure of proper equilibrium to rule out unreasonable choices in many games. One well-known example is presented later in this section.

I believe that the analysis of Section 2 provides the kind of theory that is required to determine what "reasonable doubts" players can rationally entertain regarding the choices of their opponents. For each game, rationalizability distinguishes those strategies that players could employ without violating the implications of the common knowledge they possess, from those that are patently unreasonable. If the condition that players do not take unnecessary risks is to be imposed by requiring that their conjectures give positive weight to all "likely" alternatives, those strategies that are not rationalizable should still be given zero weight. This constraint can be imposed by modifying the iterative procedure of the previous section, using the idea of a "cautious response."

Definition 6: Let $A^{i} \subseteq M^{i}$ and $X^{j} \subseteq M^{j}, j=1, \ldots, N$. A strategy $c \in A^{i}$ is 
a cautious response in $A^{i}$ to $\left(X^{1}, \ldots, X^{N}\right)$ if $\exists\left(\gamma^{1}, \ldots, \gamma^{N}\right) \in \prod_{r=1}^{N} \bar{X}^{r}$ such that (i) $\gamma^{k}$ gives positive weight to each pure strategy in $X^{k}, \forall k$; (ii) $c$ is a best response in $A^{i}$ to $\left(\gamma^{1}, \ldots, \gamma^{N}\right)$.

Definition 7: Given the sets $R^{1}(M), \ldots, R^{N}(M)$ of rationalizable strategies, for each $i$ let

$$
\begin{aligned}
C^{i}(1)= & \left\{\alpha \in R^{i}(M): \alpha \text { is a cautious response in } R^{i}(M)\right. \\
& \text { to } \left.\left(R^{1}(M), \ldots, R^{N}(M)\right)\right\} .
\end{aligned}
$$

For $t>1$, define $C^{i}(t)$ recursively for each $i$ by

$$
\begin{aligned}
& C^{i}(t)=\left\{\alpha \in R^{i}(C(t-1)): \alpha \text { is a cautious response in } R^{i}(C(t-1))\right. \\
&\text { to } \left.\left(R^{1}(C(t-1)), \ldots, R^{N}(C(t-1))\right)\right\}
\end{aligned}
$$

where $C(t-1)=\left(C^{1}(t-1), \ldots, C^{N}(t-1)\right)$, and the functions $R^{i}$ are those of Definition 1, Section 2. For each $i$,

$$
Q^{i}=\bigcap_{t=1}^{\infty} C^{i}(t)
$$

is the set of cautiously rationalizable strategies for player $i$. A profile $\left(\alpha^{1}\right.$, $\ldots, \alpha^{N}$ ) is cautiously rationalizable if $\alpha^{i} \in Q^{i} \forall i$. At each "round," strategies that are not best responses are eliminated first, and then those that are not cautious responses are removed.

Proposition 3: For some integer $k$,

$$
C^{i}(t)=C^{i}(k) \quad \forall t \geq k, \quad \forall i .
$$

Moreover, the set $Q^{i}$ of cautiously rationalizable strategies is nonempty, closed, and satisfies the pure strategy property $\forall i$.

The proof of Proposition 3 is omitted, since it is similar to those of Propositions 1 and 4. Lemma 4 of Appendix B relates the operation $C$ to weak dominance.

The solution concept performs as desired on Myerson's example $G_{2}$, and the reader can easily verify that cautious rationalizability is equally appropriate when applied to another example (not given here) constructed in Myerson [16], for which proper equilibrium also does well. But consider $G_{3}$, the normal form of a well-known extensive form game (to be called $\Gamma_{2}$ ) that is discussed in the next section. Notice that $\left(\alpha_{1}, \beta_{2}\right)$ is one of the Nash equilibrium profiles of this game; in fact, one can show $\left(\alpha_{1}, \beta_{2}\right)$ is both a trembling hand perfect, and a proper equilibrium. Why would 2 ever select $\beta_{2}$ ? $\beta_{2}$ is preferable to $\beta_{1}$ only if 1 gives considerable weight to $\alpha_{3}$. But 2 knows that $\alpha_{3}$ is strongly dominated for 1 by $\alpha_{1}$, and will never be played. Thus, there is no risk to playing $\beta_{1}$, and a superior 


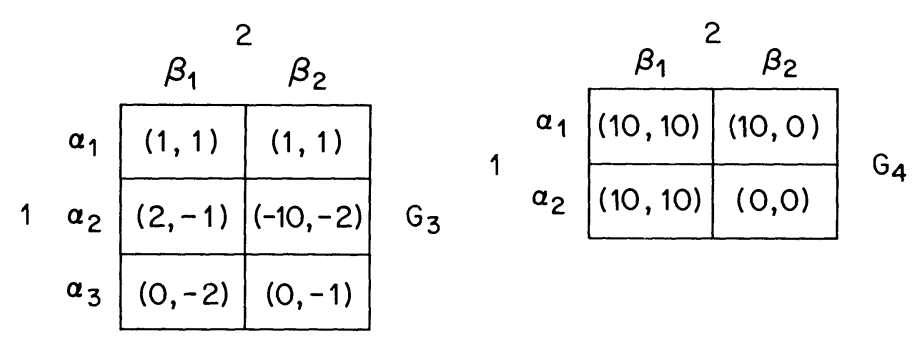

return for playing $\beta_{1}$ rather than $\beta_{2}$ if $\alpha_{2}$ is played. If 2 were a "cautious" player, it would be ridiculous for him to play $\beta_{2}$; knowing this, 1 plays $\alpha_{2}$. In the notation developed above, $C^{1}(1)$ contains all strategies giving zero weight to $\alpha_{3}$, while $C^{2}(1)=\left\{\beta_{1}\right\}$. Then $R^{1}(C(1))=\left\{\alpha_{2}\right\}$, and $R^{2}(C(1))=\left\{\beta_{1}\right\}$. No further reduction can take place; the unique cautiously rationalizable profile coincides with the only reasonable Nash equilibrium of $G_{3}$, namely $\left(\alpha_{2} ; \beta_{1}\right)$.

On the other hand, cautious rationalizability was formulated with games such as $G_{4}$ in mind, where it singles out $\beta_{1}$ for 2, but respects 1's legitimate indifference between $\alpha_{1}$ and $\alpha_{2}$ (given that 2's rationality is common knowledge, 1 knows that $\beta_{2}$ will not be played).

Bernheim's "perfect rationalizability" [2] is the natural extension of the "trembling hand" idea from Nash equilibrium to rationalizability. It is not equivalent to cautious rationalizability, which is motivated quite differently. In $G_{3}$, for example, $\beta_{2}$ is perfectly, but not cautiously, rationalizable. Conversely, in $G_{4}, \alpha_{2}$ is cautiously, but not perfectly, rationalizable.

\section{RATIONALIZABILITY IN THE EXTENSIVE FORM}

This section generalizes the analysis of Section 2 to games having some sequential nature. In this context it is possible to study the best-known type of imperfect behavior, namely unreasonable behavior at unreached information sets. The problem is attacked using the idea of consistent conjectures, without the additional assumptions needed to ensure cautious behavior. Those assumptions are invoked in Section 5, because what I have called imperfections of the second type may still arise in the extensive form.

A complete formal description of an extensive form game would be too lengthy to be appropriate here. Some knowledge of extensive form games and their normal forms is taken for granted, but a number of initial definitions are unavoidable. The reader who requires precise definitions of the terms used here should consult Selten [21].

I restrict myself to finite $N$-person extensive form games of perfect recall (Kuhn [13]). At the beginning of the game $\Gamma$, "nature" makes a (possibly degenerate) random move. ${ }^{4} I^{i j}$ denotes the $j$ th information set of the $i$ th player,

\footnotetext{
${ }^{4}$ Harsanyi [10] has shown that games having various sorts of incomplete information, such as incomplete knowledge of others' utility functions, can be handled by an ingenious use of the random move at the beginning of the game. Hence the solution concept defined here encompasses such situations.
} 
and $S^{i j}$ the set of choices at $I^{i j}$. For $j \neq k, I^{i j}$ is a predecessor of $I^{i k}$ if there exist a terminal node $y$ and a node $x$ in $I^{i j}$ such that the path from $x$ to $y$ goes through $I^{i k} ; I^{i k}$ is a successor of $I^{i j}$. A pure strategy $f$ for player $i$ is a function associating with each information set $I^{i j}$ of $i$ one of the choices in $S^{i j}$; denote this choice by $f(i, j)$.

DEFINITION 8: If $f$ and $g$ are pure strategies for $i, g$ is an $i j$-replacement for $f$ if for all $l \neq j$ such that $I^{i l}$ is not a successor to $I^{i j}, g(i, l)=f(i, l)$.

This says that $f$ and $g$ agree everywhere except on $I^{i j}$ and its successors.

With each profile of pure strategies is associated a utility for each player; the domain of the utility functions $U^{i}$ is extended to $\prod_{r=1}^{N} M^{r}$ by an expected utility calculation, where $M^{r}$ is the mixed strategy simplex of player $r$. Consider a particular information set $I^{i j}$ and a profile $m=\left(m^{1}, \ldots, m^{N}\right)$ of (mixed) strategies. If for each terminal node $y$ reached with positive probability when $m$ is played, and each $x \in I^{i j}, x$ does not lie on the path from the origin to $y$, then $I^{i j}$ is not reached by $m$. If the condition is violated, $I^{i j}$ is reached by $m$.

Consider the game $\Gamma_{1}$ having perfect information (all information sets are singletons) and no randomness. (When representing games where the random move is restricted to one choice, I simply omit the random player's information set.) Although the outcome yielding $(0,0)$ is absurd, it is among the Nash equilibrium outcomes of $\Gamma_{1}$. If 1 specifies the choice $\alpha_{1}$ (with probability 1) and 2 chooses $\beta_{2}$, neither has an incentive to deviate. But everyone must agree that if 1 were to play $\alpha_{2} 2$ would, upon being reached, respond by playing $\beta_{1}$. Knowing this, 1 should play $\alpha_{2}$. The imperfect behavior arises because in the dubious equilibrium, 2's information set is not reached with positive probability. Consequently 2 can specify any choice with impunity.

Subgame perfect equilibrium (Selten [20, 21]) deals nicely with examples of this variety. A Nash equilibrium is subgame perfect if the strategies it induces on any proper subgame of $\Gamma$ (see Selten [21]) constitute a Nash equilibrium of that subgame. In $\Gamma_{1}, 2$ 's choice of $\beta_{2}$ is not Nash on the subgame starting at 2's information set.

Unfortunately there are often too few proper subgames to allow subgame perfection to enforce intuitively reasonable behavior in a game. This prompted Selten [21] to introduce a further notion, perfect equilibrium, or trembling hand

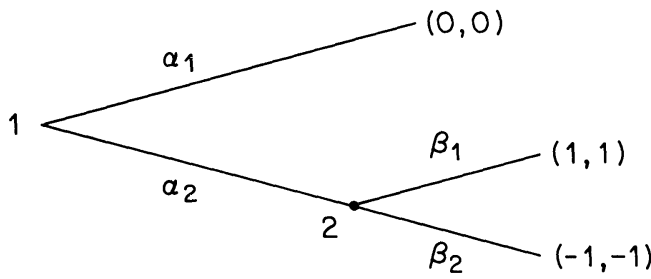


perfect equilibrium. The set of perfect equilibria is a subset of the set of subgame perfect equilibria. As was noted in Section 3, the indiscriminate nature of the "trembles" allowed causes problems for the perfect equilibrium concept. The attempt by Myerson [16] to correct this by limiting the class of admissible trembles was only partially successful; proper equilibrium remains too deeply rooted in the stochastic "small mistakes" framework to escape all the difficulties created by that approach. A major alternative has been suggested by Kreps and Wilson [12]. Their solution concept, sequential equilibrium, is based upon an examination of rational beliefs rather than the possibilities for error.

While all of the solution concepts mentioned above have features that are extremely attractive, examples abound in which none of the equilibrium notions is satisfactory (one well-known example is presented later in this section). Equally important is the fact that they all admit Nash profiles only; this paper attempts to escape that restriction. Let us try to apply the idea of consistent conjectures to examples such as $\Gamma_{1}$.

The possibility of collapsing series of choices into timeless contingent strategies must not obscure the fact that the phenomenon being modelled is some sequential game, in which conjectures may be contradicted in the course of play. In $\Gamma_{1}$, it is ludicrous to maintain that if 2 is called upon to move, having been reached, he might choose $\beta_{2}$, thinking that $\alpha_{1}$ was played by 1 . By the time he must commit himself to a course of action, 2 knows that it is a fact that 1 played $\alpha_{2}$. The observation that a conjecture must not be maintained in the face of evidence that refutes it is a central element of the sequential equilibrium concept; it is combined here with a further principle and the iterative techniques of previous sections to construct a new solution concept for extensive form games.

Since a player's beliefs about others' strategies may be refuted as a play of the game progresses, he might need to formulate new conjectures as the old ones are disproven. Consequently I associate a conjecture

$$
c^{i j}=\left(c^{i j}(1), \ldots, c^{i j}(N)\right)
$$

with each information set $I^{i j}$ in $\Gamma ; c^{i j}(k)$ represents what an "agent" $j$ for player $i$ believes, once $I^{i j}$ is reached, about what player $k$ 's mixed strategy is. A conjecture $c^{i j}(k)$ over a set $A^{k} \subseteq M^{k}$ can be regarded as an element of $\bar{A}^{k}$ (see Appendix A).

I have noted that an agent $i j$, upon being reached, should not entertain a conjecture that does not reach $I^{i j}$. A further restriction, not invoked in other solution concepts, is appropriate: if the information set can be reached without violating the rationality of any player, then the agent's conjecture must not attribute an irrational strategy to any player. In other words, he should seek a reasonable explanation for what he has observed. This principle is applied within an iterative procedure similar to that of Section 2, suitably elaborated to exploit the additional information in the extensive form.

For later reference, the iterative procedure is defined for sets $H^{1}, \ldots, H^{N}$ satisfying certain properties; our immediate interest is in the technique applied to $M=\left(M^{1}, \ldots, M^{N}\right)$. 
Definition 9: Let $H=\left(H^{1}, \ldots, H^{N}\right)$ where $\forall i H^{i}$ is closed, nonempty, and has the pure strategy property. Define $H^{i}(0)=H^{i}, i=1, \ldots, N$. For any $t \geq 1$, define the sets $H^{1}(t), \ldots, H^{N}(t)$ recursively as follows. For each pure strategy $\beta \in H^{i}(t-1)$, let $J^{i}(\beta, H, t)$ contain all those $j$ such that $I^{i j}$ can be reached by some profile of the form $\left(m^{1}, \ldots, m^{i-1}, \beta, m^{i+1}, \ldots, m^{N}\right)$, where $m^{r} \in$ $H^{r}(t-1), r=1, \ldots, N$. (The eventual interpretation will be that at stage $t$ of the logical deduction process, $i$ knows that if he plays $\beta$, no information set $I^{i j}$ will be reached unless $j \in J^{i}(\beta, H, t)$.) A strategy $\alpha \in H^{i}(t-1)$ giving positive weight to pure strategies $\alpha_{1}, \ldots, \alpha_{h}$ is an element of $H^{i}(t)$ if there exist conjectures $c_{z}^{i j}, z=1, \ldots, h$, such that for all $z$, and all $j \in J^{i}\left(\alpha_{z}, H, t\right)$ :

(i) $c_{z}^{i j}(i)=\alpha_{z}$;

(ii) $c_{z}^{i j}(l)=c_{1}^{i j}(l), l \neq i$;

(iii) for $r, s \in J^{i}\left(\alpha_{z}, H, t\right)$, if $I^{i r}$ is a predecessor of $I^{i s}$ and $c_{z}^{i r}$ reaches $I^{i s}$, then $c_{z}^{i s}=c_{z}^{i r}$

(iv) $c_{z}^{i j}$ reaches $I^{i j}$;

(v) $c_{z}^{i j} \in \prod_{r=1}^{N} \bar{H}^{r}(t-1)$; and

(vi) $\alpha_{z}$ is a best response to $c_{z}^{i j}$ among all ij-replacements for $\alpha_{z}$ in $H^{i}(t-1)$.

For each $i$, define

$$
R^{i}(H)=\bigcap_{t=1}^{\infty} H^{i}(t) .
$$

Definition 10: $R^{i}(M)$ is the set of rationalizable strategies for player $i$, where $M=\left(M^{1}, \ldots, M^{N}\right)$ is the vector of mixed strategy sets.

The iterative procedure is interpreted as follows. At each stage, additional restrictions are placed on conjectures and actions only at information sets that can be reached by profiles of strategies not previously eliminated. In a particular play of the game, player $i$ uses some pure strategy $\alpha_{z}$ which is a realization of the mixed strategy $\alpha$. Condition (i) says that $i$ 's "conjecture" about his own strategy is correct. The next requirement stipulates that conjectures about others' strategies do not depend upon which of the $\alpha_{1}, \ldots, \alpha_{h}$ player $i$ ends up using. According to (iii), a conjecture should not be discarded unless it is contradicted (by arrival at an information set unreachable by the conjecture in question). Condition (iv) ensures that a conjecture at $I^{i j}$ explains how that information set could have been reached. The principle that the explanation should be "reasonable" is embodied in (v), which restricts conjectures to strategies that have not been eliminated at a previous stage. Finally, the strategy chosen by $i$ should at all times be an optimal response to the conjectures he holds. The most convenient way to express this condition is to consider $i j$-replacements for $\alpha_{z}$; these represent the options still open to $i$ at $I^{i j}$. Among these, $\alpha_{z}$ must constitute an optimal contingent plan, given that beliefs about others' mixed strategies are described by $c_{z}^{i j}$. 
Proposition 4: Under the assumptions of Definition 9, for all $i$ and $t, H^{i}(t)$ is nonempty, closed, and has the pure strategy property. Furthermore $\exists k$ such that $\forall i$, $H^{i}(t)=H^{i}(k), \forall t \geq k$.

Proof: The sets $H^{i}(t), i=1, \ldots, N$, inherit the pure strategy property, nonemptiness, and closedness from the original sets $H^{i}$. This is easy to see in the case of the pure strategy property, because if the pure strategies of which a mixed strategy $\alpha$ is comprised can collectively satisfy (i) to (vi), each of the pure strategies satisfies the conditions individually. To show nonemptiness, assume $H^{1}(t-1), \ldots, H^{N}(t-1)$ are nonempty and closed, and choose any conjecture $\tilde{c}=(\tilde{c}(1), \ldots, \tilde{c}(N))$ such that $\tilde{c}(r) \in \bar{H}^{r}(t-1)$ gives positive weight to every pure strategy in $H^{r}(t-1)$. Since $U^{i}$ is continuous and $H^{i}(t-1)$ is nonempty and compact, there exists an $\alpha$ that is a best response in $H^{i}(t-1)$ to $\tilde{c} . \alpha$ may be chosen to be a pure strategy, because $H^{i}(t-1)$ has the pure strategy property. For every $j \in J^{i}(\alpha, H, t)$, define

$$
c_{1}^{i j}=(\tilde{c}(1), \ldots, \tilde{c}(i-1), \alpha, \tilde{c}(i+1), \ldots, \tilde{c}(N)) .
$$

$\alpha$ and $c_{1}^{i j}$ satisfy (i) to (vi). (i) holds by definition. (ii) is trivially satisfied because there is only one pure strategy involved. (iii) is equally clear since $c_{j}^{i j}$ is not a function of $j$ as defined. In all components except $i, c_{j}^{i j}$ gives positive weight to all pure strategies not eliminated in previous rounds; hence $c_{1}^{i j}$ reaches $I^{i j}$ for all $j \in J^{i}(\alpha, H, t)$, and (iv) is satisfied. (v) holds by the definition of $\tilde{c}$. Since $\alpha$ is a best response to $c_{1}^{i j}$ in $H^{i}(t-1), \alpha$ is certainly a best response to $c_{1}^{i j}$ in the set of all $i j$-replacements for $\alpha$ in $H^{i}(t-1)$; therefore $\alpha \in H^{i}(t)$. To establish that $H^{i}(t)$ is closed, consider a sequence $\beta_{1}, \beta_{2}, \ldots$ in $H^{i}(t)$ converging to a strategy $\beta$. $H^{i}(t-1)$ is closed by hypothesis, so $\beta \in H^{i}(t-1)$. For some integer $V$, it must be the case that for all $W \geq V, \beta_{W}$ gives positive weight to (at least) all the pure strategies given positive weight by $\beta$. But there exists a set of conjectures $c_{z}^{i j}$ (where $z$ indexes the pure strategies comprising $\beta_{V}$ ) such that $\beta_{V}$ and the $c_{z}^{i j}$ satisfy (i) to (vi). Then $\beta$ and the $c_{z}^{i j}$ (omitting any conjecture corresponding to pure strategies not given positive weight by $\beta$ ) satisfy (i) to (vi). Thus $\beta \in H^{i}(t)$, and the set is closed.

$H^{i}(t+1)$ can differ from $H^{i}(t)$ only if for some $j, \bar{H}^{j}(t) \neq \bar{H}^{j}(t-1)$. But since $H^{j}(t)$ and $H^{j}(t-1)$ both satisfy the pure strategy property, their convex hulls differ only if some pure strategy in $H^{j}(t-1)$ is absent from $H^{j}(t)$. Thus, the iterative procedure "stops" in $k$ steps for some finite $k$, because pure strategies are in finite supply.

COROLlARY: The rationalizable sets $R^{1}(M), \ldots, R^{N}(M)$ are nonempty, closed, and satisfy the pure strategy property. Thus a rationalizable profile of pure strategies always exists.

Proof: Set $H^{i}=M^{i} \forall i$ in Proposition 10 . 


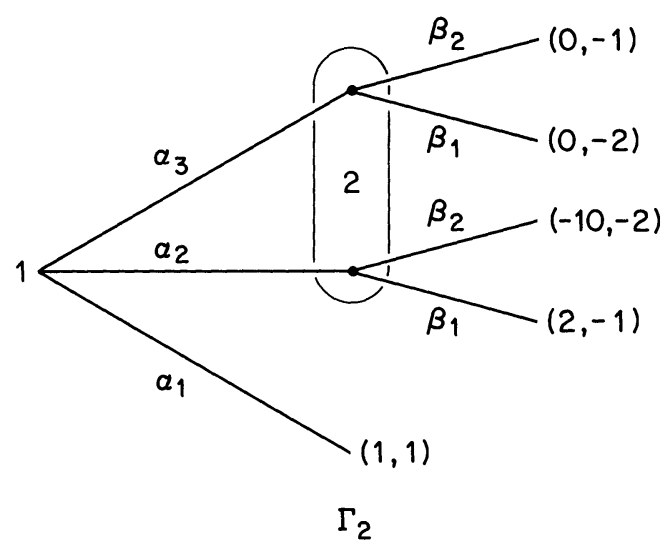

To get some feeling for how this solution concept operates, consider two examples, starting with the familiar $\Gamma_{1}$. In that game, 1 is unable to eliminate any strategy in the first round. Since strategies of 1 that reach 2's information set must give positive weight to $\alpha_{2}, 2$ must remove all strategies that are not best responses to some such strategy. This eliminates all strategies of 2 except $\beta_{1}$, so in the next round, 1 retains the only strategy that is a best response to $\beta_{1}$, namely $\alpha_{2}$.

A more challenging test for the theory is an example that Kreps and Wilson [12] attribute to E. Kohlberg. (The example is robust: small perturbations in the payoffs will not alter any of the statements made below.) In the game $\Gamma_{2}$, player 2 has only one information set, which is indicated in the game tree by enclosing the two nodes in that information set by an oblong figure. Notice that $\alpha_{1}$ strongly dominates $\alpha_{3}$; the latter will never be played with positive probability by a rational player. If reached, 2 should conclude that $\alpha_{2}$ was played and respond optimally by playing $\beta_{1}$. Knowing that this would be 2 's response, 1 should play $\alpha_{2}$. Despite this simple argument, another Nash equilibrium (which can actually be shown to be a trembling hand perfect, proper, and sequential equilibrium) has 1 playing $\alpha_{1}$ with certainty and 2 playing $\beta_{2}$. This is not rationalizable. In the first "round," all strategies giving $\alpha_{3}$ positive weight are removed. In the second round, since these strategies are absent from $M^{1}(1), 2$ eliminates every strategy except $\beta_{1}$, because elements of $M^{1}(1)$ reaching 2's information set are those giving some positive weight to $\alpha_{2}$. In the third round, 1 has a unique best response $\alpha_{2}$ to the single element $\beta_{1}$ in $M^{2}(2)$. The only rationalizable profile of $\Gamma_{2}$ is what Kreps and Wilson agree is the only reasonable profile. Their general remarks on what beliefs should be admissible are interesting:

\footnotetext{
"Some sequential equilibria are supported by beliefs that the analyst can reject because they are supported by beliefs that are implausible. We will not propose any formal criteria for 'plausible beliefs' here. In certain cases, such as Myerson's concept of properness, some formalization is possible. In other cases, it is not clear that any formal criteria can be devised-it may be that arguments must be tailored to the particular game" (Kreps and Wilson [12, p. 885]).
} 
Rationalizability formalizes the notion that beliefs may be implausible at an information set because (i) the set could not have been reached had those beliefs been true, or (ii) they are inconsistent with the results of logical deductions based on what players know about one another and the rules of the game. If rationalizability fails to narrow down the possible outcomes significantly in a given game, one might then consider applying criteria of a more ad hoc description, and perhaps make predictions on a game-by-game basis as Kreps and Wilson suggest.

\section{CAUTIOUS RATIONALIZABILITY IN THE EXTENSIVE FORM}

It is straightforward to verify that in a perfectly simultaneous nonstochastic game, the rationalizable sets conform to the normal form definition given in Section 2, applied to the normal form of the game in question. But in such games, rationalizable behavior is not always "cautious": the solution concept does not prevent imperfection of the second type. A simple demonstration that this applies equally to the extensive form is given by $\Gamma_{3}$, whose normal form is $G_{2}$, Myerson's example. If both players make prudent choices, $\left(\alpha_{1} ; \beta_{1}\right)$ will result. But $\left(\alpha_{2} ; \beta_{2}\right)$ is also rationalizable. Such behavior can be avoided by the same technique as that employed in Section 3. A natural generalization of the normal form analysis is accomplished here as briefly as possible.

Definition 11: Given the sets $R^{1}(M), \ldots, R^{N}(M)$ of (extensive form) rationalizable strategies, for each $i$ define

$$
\begin{aligned}
& C^{i}(1)=\left\{\alpha \in R^{i}(M): \alpha \text { is a cautious response in } R^{i}(M)\right. \\
&\text { to } \left.\left(R^{1}(M), \ldots, R^{N}(M)\right)\right\} .
\end{aligned}
$$

For $t>1$, define $C^{i}(t)$ recursively for each $i$ by

$$
\begin{aligned}
& C^{i}(t)=\left\{\alpha \in R^{i}(C(t-1)): \alpha \text { is a cautious response in } R^{i}(C(t-1))\right. \\
&\text { to } \left.\left(R^{1}(C(t-1)), \ldots, R^{N}(C(t-1))\right)\right\}
\end{aligned}
$$

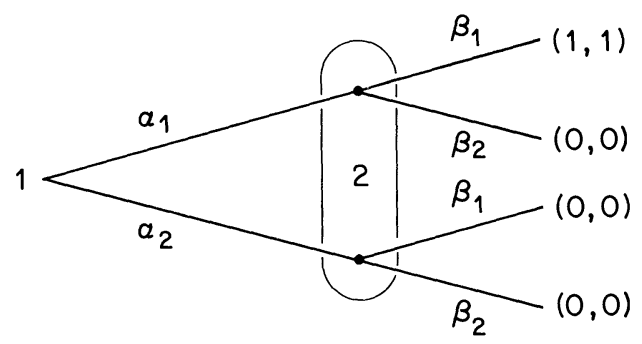

$\Gamma_{3}$ 
where $C(t-1)=\left(C^{1}(t-1), \ldots, C^{N}(t-1)\right)$, and the functions $R^{i}$ are those of Definition 10, Section 2. For each $i$,

$$
Q^{i}=\bigcap_{t=1}^{\infty} C^{i}(t)
$$

is the set of cautiously rationalizable strategies for player $i$. A profile $\left(\alpha^{1}\right.$, $\ldots, \alpha^{N}$ ) is cautiously rationalizable if $\alpha^{i} \in Q^{i} \forall i$.

At each "round," strategies that are not best responses are discarded first, and then those that are not cautious responses are removed.

Proposition 5: For some integer $k$,

$$
C^{i}(t)=C^{i}(k) \quad \forall t \geq k, \quad \forall i .
$$

Moreover, the set $Q^{i}$ of cautiously rationalizable strategies is nonempty, closed, and satisfies the pure strategy property $\forall i$.

The proof is a straightforward extension of the proof of Proposition 4, and is omitted.

The solution concept has the attractive feature that in the play of a game, no one's conjectures are ever contradicted. Since each person's conjecture gives positive weight to every cautiously rationalizable strategy of every other player, nothing that is believed by any player to have zero probability ever occurs, so long as others choose cautiously.

It might appear at first glance that in a game such as $\Gamma_{4}$ in which 1 should be indifferent between $\alpha_{1}$ and $\alpha_{2}$ (according to subgame perfection or backward induction), cautious rationalizability forces 1 to choose $\alpha_{1}$, by eliminating $\alpha_{2}$ in the first round, before $\beta_{2}$ has been removed. In fact this does not happen. Recall that before the cautious response criterion comes into play, the rationalizable sets are calculated. For 2, this eliminates all strategies except $\beta_{1}$; in "cautious response" to this, 1 plays either $\alpha_{1}$ or $\alpha_{2}$.

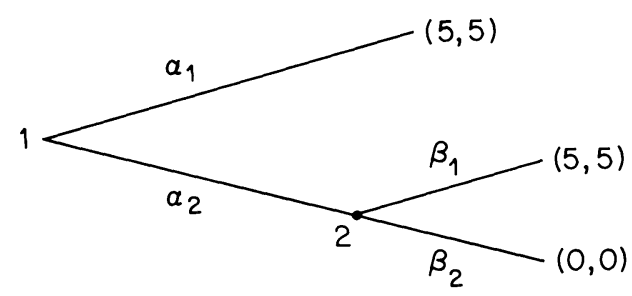

$\Gamma_{4}$ 


\section{CONCLUSION}

In response to the opening question: "What constitutes rational behavior in a noncooperative strategic situation?" an extremely conservative theory of strategic behavior, rationalizability, has been developed. Without attempting to predict behavior uniquely in all games, the solution concept rules out strategic choices on the basis of rather fundamental principles such as maximization of expected utility, and the common knowledge assumption. Rationalizability is well suited to dealing with implausible behavior at "unreached" information sets, but an additional assumption that players are in some sense cautious is needed to deal with a second kind of imperfection. Incorporation of this assumption results in a more restrictive solution concept, cautious rationalizability.

In conclusion, I wish to emphasize two points. First, as a necessary condition for a strategy profile to be reconcilable with the rationality of the players, the appropriate criterion is rationalizability rather than Nash equilibrium. Secondly, when one analyzes an economic or abstract game, every attempt should be made to exploit the informational structure of the extensive form, whether the objective is to make a specific prediction, or simply to place bounds upon what outcomes could possibly arise.

\section{Princeton University}

Manuscript received June, 1982; final revision received June, 1983.

\section{APPENDIX A}

A conjecture over a set $A$ in Euclidean space is a probability measure $\mu$ defined on the Borel sets of $A$. A trivial corollary of Lemma 1 below is that the mean of $A$ with respect to $\mu$ lies in $\bar{A}$, the convex hull of $A$. Lemma 2 states that the expected utility associated with the conjecture $\mu$ can be calculated using the mixed strategy $y=\int_{A} x \mu(d x)$.

Lemma 1: Suppose that $A$ is a convex subset of Euclidean space, and $\mu(A)=1$.

$$
y \equiv \int_{A} x \mu(d x) \in A .
$$

Proof: If $\mu$ is a point mass, the result is immediate. If not, find a minimal affine subspace $S$ such that $\mu(A \cap S)=1$. Without loss of generality assume $0 \in S$. If $y \in S \backslash A, \exists p \in S, p \neq 0$ such that $\sup p \cdot(A \cap S) \leq p \cdot y$. The set $S^{\prime}=\{x \in S: p \cdot x=\sup p \cdot(A \cap S)\}$ has lower dimension than $S$, and hence $\mu\left(A \cap S^{\prime}\right)<1$. Thus $\int_{A} \sup p \cdot(A \cap S) \mu(d x)>\int_{A} p \cdot x \mu(d x)$.

Now

$$
\begin{aligned}
\sup p \cdot(A \cap S) & =\int_{A}(\sup p \cdot(A \cap S)) \mu(d x) \\
& >\int_{A} p \cdot x \mu(d x) \\
& =p \cdot \int_{A} x \mu(d x) \\
& =p \cdot y, \quad \text { a contradiction. }
\end{aligned}
$$


Lemma 2: Let $M$ be the mixed strategy simplex associated with the pure strategy set $S=$ $\left\{\alpha_{1}, \ldots, \alpha_{w}\right\}$, and let $U: M \rightarrow \mathbb{R}$. Let $y=\int_{M} x \mu(d x)$, where $\mu(M)=1$. Then

$$
\int_{M}\left(\sum_{i=1}^{w} x_{i} U\left(\alpha_{i}\right)\right) \mu(d x)=\sum_{i=1}^{w} y_{i} U\left(\alpha_{i}\right)
$$

ProOF:

$$
\begin{aligned}
\int_{M}\left(\sum_{i=1}^{w} x_{i} U\left(\alpha_{i}\right)\right) \mu(d x) & =\sum_{i=1}^{w} \int_{M} x_{i} U\left(\alpha_{i}\right) \mu(d x) \\
& =\sum_{i=1}^{w} U\left(\alpha_{i}\right) \int_{M} x_{i} \mu(d x) \\
& =\sum_{i=1}^{w} y_{i} U\left(\alpha_{i}\right) .
\end{aligned}
$$

\section{APPENDIX B}

This appendix presents two lemmas relating the properties "best response" and "cautious response," to strong and weak dominance, respectively. Related results have been established in the literature ${ }^{5}$ (see, for example, Ferguson [7, Theorem 1, p. 86]) but the proofs are included here for completeness. Dilip Abreu suggested the arguments used below. Note that the results are not restricted to zero-sum games, but cannot be generalized to $N$-person games, where the propositions are false. However if one permits opponents to correlate their random strategies, the proofs are easily extended to the $N$-person case.

LeMMA 3: Let $G=\left(S^{1}, S^{2} ; U^{1}, U^{2}\right)$ be a finite noncooperative game, with associated mixed strategy sets $M^{1}$ and $M^{2} . \alpha \in M^{1}$ is strongly dominated if and only if $\exists m \in M^{2}$ such that $\alpha$ is a best response to $m$.

Proof: If some $\beta \in M^{1}$ strongly dominates $\alpha$, then $\forall \gamma \in M^{2}, U^{1}(\beta, \gamma)>U^{1}(\alpha, \gamma)$, so $\alpha$ is never a best response. To establish the converse, suppose $\alpha$ is not a best response to any element of $M^{2}$. Then there exists a function $b: M^{2} \rightarrow M^{1}$ with $U^{1}(b(m), m)>U^{1}(\alpha, m) \forall m$. Consider the zero-sum game $\bar{G}=\left(S^{1}, S^{2} ; \bar{U}^{1}, \bar{U}^{2}\right)$ where $\bar{U}^{1}(x, y)=U^{1}(x, y)-U^{1}(\alpha, y)$ and $\bar{U}^{2}(x, y)=-\bar{U}^{1}(x, y)$. Let $\left(x^{*}, y^{*}\right)$ be a Nash equilibrium of $\bar{G}$. For any $m \in M^{2}$,

$$
\begin{aligned}
\bar{U}^{1}\left(x^{*}, m\right) & \geq \bar{U}^{1}\left(x^{*}, y^{*}\right) \\
& \geq \bar{U}^{1}\left(b\left(y^{*}\right), y^{*}\right) \\
& >\bar{U}^{1}\left(\alpha, y^{*}\right) \\
& =0 .
\end{aligned}
$$

But

$$
\begin{aligned}
& \bar{U}^{1}\left(x^{*}, m\right)>0 \\
& \forall m \Rightarrow U^{1}\left(x^{*}, m\right)>U^{1}(\alpha, m) \quad \forall m
\end{aligned}
$$

$\therefore \alpha$ is strongly dominated by $x^{*}$.

Q.E.D.

${ }^{5}$ After the page proofs of this paper were prepared, I learned from Eric van Damme that Lemmas 3 and 4 are extremely closely related to Lemma 3.2.1 and Theorem 3.2.2 in van Damme [4] and to much earlier work of Gale and Sherman [9]. 
Because the derivation of cautiously rationalizable strategies involves alternation of the operations $R$ and $C$ (see Section 3), this criterion differs from "iterative weak dominance" techniques, as $G_{4}$ of Section 3 illustrates. Lemma 4 shows, however, that there is a close connection between "caution" and weak dominance.

LEMMA 4: Let $G=\left(S^{1}, S^{2} ; U^{1}, U^{2}\right)$ be a finite noncooperative game, with associated mixed strategy sets $M^{1}$ and $M^{2} . \alpha \in M^{1}$ is weakly dominated if and only if $\alpha$ is not a cautious response to $\left(M^{1}, M^{2}\right)$.

Proof: Suppose that $\alpha$ is weakly dominated by some $\gamma \in M^{1}$. Then for any $x \in M^{2}$ giving strictly positive weight to every pure strategy in $M^{2}, U^{1}(\alpha, x)<U^{1}(\gamma, x)$, so by definition $\alpha$ is not a cautious response to $\left(M^{1}, M^{2}\right)$.

To establish the converse, suppose that $\alpha$ is not a cautious response. Define

$$
A=\left\{\alpha^{\prime} \in M^{1}: U^{1}\left(\alpha^{\prime}, x\right)=U^{1}(\alpha, x) \forall x \in M^{2}\right\} .
$$

Let $k$ be the number of pure strategies in $M^{2}$, and $T$ be the open interval $(0,1 / k)$. Define

$$
\begin{aligned}
& \delta_{\epsilon}=\left\{x \in M^{2}: x_{i} \geq \epsilon, i=1, \ldots, k\right\}, \\
& B_{\epsilon}=\left\{\beta \in M^{1}: U^{1}(\beta, x)>U^{1}(\alpha, x) \forall x \in \delta_{\epsilon}\right\}, \\
& W_{\epsilon}=\left\{\beta \in M^{1}: U^{1}(\beta, x) \geq U^{1}(\alpha, x) \forall x \in \delta_{\epsilon}\right\} .
\end{aligned}
$$

$\alpha$ is not a cautious response to $\left(M^{1}, M^{2}\right)$, so for each $\epsilon \in T, \alpha$ is not a best response to any $x \in \delta_{\epsilon}$, and a repetition of the argument of Lemma 3 (regarding $\delta_{\epsilon}$ as the opponent's strategy space) establishes that $B_{\epsilon}$ is nonempty. Since $W_{\epsilon}$ is closed and nonempty, for each $\epsilon \in T$ we can choose $\beta_{\epsilon} \in M^{1}$ that is a best response in $W_{\epsilon}$ to $(1 / k, \ldots, 1 / k) \in \delta_{\epsilon}$. Notice that $\beta_{\epsilon}$ yields 1 strictly higher utility against $(1 / k, \ldots, 1 / k)$ than $\alpha$, since $B_{\epsilon} \subseteq W_{\epsilon}$. Choose a sequence of $\epsilon_{i}$ 's in $T$ converging to 0 , such that $\left\{\beta_{\epsilon}\right\}$ converges; let $\beta^{*}$ be the limit of the sequence $\left\{\beta_{\epsilon_{1}}\right\}$. We will show that $\beta^{*}$ weakly dominates $\alpha$.

Continuity of $U^{1}$ guarantees that $\beta^{*}$ is at least as good for 1 as $\alpha$ against all $x \in M^{2}$. It remains only to show that $\beta^{*} \notin A$. If $\exists \alpha^{\prime} \in A$ with $\alpha^{\prime}=\beta^{*}$, then for all sufficiently small $\epsilon_{i}, \beta_{\epsilon}$ gives positive weight to every pure strategy given positive weight by $\alpha^{\prime}$. Then $\lambda>0$ can be chosen sufficiently small so that all components of

$$
\bar{\beta}_{\epsilon_{1}}=\left(\beta_{\epsilon_{1}}-\lambda \alpha^{\prime}\right) \frac{1}{1-\lambda}
$$

are nonnegative. For any $x \in \delta_{\epsilon}$,

$$
U^{1}\left(\bar{\beta}_{\epsilon}, x\right)-U^{1}\left(\beta_{\epsilon}, x\right)=\frac{\lambda}{1-\lambda}\left[U^{1}\left(\beta_{\epsilon}, x\right)-U^{1}(\alpha, x)\right] \geq 0
$$

because $\beta_{\epsilon_{\epsilon}} \in W_{\epsilon_{1}}$. Moreover the inequality is strict when $x=(1 / k, \ldots, 1 / k)$. Thus $\bar{\beta}_{\epsilon_{1}}$ is in $W_{\epsilon}$ and yields 1 higher utility than $\beta_{\epsilon}$ against $(1 / k, \ldots, 1 / k)$, a contradiction.

Q.E.D.

\section{REFERENCES}

[1] Aumann, R.: “Agreeing to Disagree," The Annals of Statistics, 4(1976), 1236-1239.

[2] Bernheim, D.: "Rationalizable Strategic Behavior," Econometrica, 52(1984), 1007-1028.

[3] Bryant, J.: "Perfection, the Infinite Horizon and Dominance," Economics Letters, 10(1982), 223-229.

[4] Damme, E. E. C. van: Refinements of the Nash Equilibrium Concept. Berlin: Springer-Verlag, 1983.

[5] Ellsberg, D.: "Theory of the Reluctant Duelist," American Economic Review, 46(1956), 909923.

[6] Farquharson, R.: Theory of Voting. New Haven: Yale University Press, 1957; 1969.

[7] Ferguson, T. S.: Mathematical Statistics. New York: Academic Press, 1967. 
Gale, D.: “A Theory of $N$-Person Games with Perfect Information," Proceedings of the National Academy of Sciences, 39(1953), 496-501.

[9] Gale, D., and S. Sherman: "Solutions of Finite Two-person Games," in Contributions to the Theory of Games, Vol. 1, ed. by H. Kuhn and A. Tucker. Princeton: Princeton University Press, 1950.

[10] HarsanYI, J. C.: "Games with Incomplete Information Played by 'Bayesian' Players, I-III," Management Science, 14(1967-1968), 159-182, 320-334, 486-502.

[11] — : "A Solution Concept for $n$-Person Noncooperative Games," International Journal of Game Theory, 5(1976), 211-225.

[12] KrePs, D., AND R. Wilson: "Sequential Equilibria," Econometrica, 50(1982), 863-894.

[13] Kunn, H. W.: "Extensive Games and the Problem of Information," in Contributions to the Theory of Games, Vol. 2, ed. by H. Kuhn and A. Tucker. Princeton: Princeton University Press, 1953.

[14] LuCE, R. D., AND H. Raiffa: Games and Decisions. New York: John Wiley and Sons, 1957.

[15] Moulin, H.: "Dominance Solvable Voting Schemes," Econometrica, 47(1979), 1337-1351.

[16] Myerson, R. B.: "Refinements of the Nash Equilibrium Concept," International Journal of Game Theory, 7(1978), 73-80.

[17] NaSh, J. F.: "Non-Cooperative Games," Annals of Mathematics, 54(1951), 286-295.

[18] Pearce, D.: "Ex Ante Equilibrium: Strategic Behaviour and the Problem of Perfection," Econometric Research Program Research Memorandum 301, Princeton University, 1982.

[19] — : "A Problem with Single-Valued Solution Concepts," unpublished manuscript, Princeton University, 1983.

[20] Selten, R.: "Spieltheoretische Behandlung eines Oligopolmodells mit Nachfrageträgheit," Zeitschrift für die Gesamte Staatswissenschaft, 121(1965), 301-324.

[21] — : "Reexamination of the Perfectness Concept for Equilibrium Points in Extensive Games," International Journal of Game Theory, 4(1975), 25-55. 